\title{
Educação médica continuada online: potencial e desafios no cenário brasileiro
}

\section{Online continuing medical education: potential and challenges in the Brazilian context}

\author{
Maria Teresa Meirelles Leite ${ }^{\mathrm{I}}$ \\ Alda Luiza Carlini ${ }^{\text {II }}$ \\ Monica Parente Ramos ${ }^{\mathrm{I}}$ \\ Daniel Sigulem ${ }^{\mathrm{I}}$
}

\section{PALAVRAS-CHAVE \\ - Educação a Distância \\ - Educação Médica Continuada \\ - Educação Médica}

\section{KEY WORDS}

- Education, Distance

- Education, Medical, Continuing

- Education, Medical
Recebido em: 17/03/2009 Reencaminhado em: 14/05/2009 Aprovado em: 30/06/2009

REVISTA BRASILEIRA DE EDUCAÇ̃̃O MÉDICA

\begin{abstract}
A B S T R A C T
With the intense production and publication of scientific information, it has become difficult for medical professionals to keep up-to-date using only the traditional resources. It is urgent to become familiar with and participate in continuing training processes. Among other initiatives, the Brazilian Medical Association and the National Medical Board launched the National Program for OnlineContinuing Medical Education, seeking to disseminate the knowledge produced in large cities to professionals in the more remote areas of the country or those with less available time. Based on the assumption that the Knowledge Society requires initial and on-going training for professionals and citizens with a new set of skills to act efficiently and responsibly, these programs should be developed through pedagogical approaches that effectively value, beyond content delivery, a willingness to engage in research, autonomy in the search for information, collaborative spirit, and an ethical stance. To contribute to this discussion, the current article aims to resume the process of formal organization of online continuing medical education in Brazil in didactic and pedagogical terms and to analyze the perspectives of distance education programs.
\end{abstract}




\section{INTRODUÇÃO}

Historicamente, a atualização profissional de médicos no Brasil tem acontecido informalmente, fora do contexto universitário ou de associações médicas. Além das eventuais participações em congressos e seminários, muitos profissionais recebem notícias técnicas e científicas apenas pela ação dos propagandistas de laboratórios farmacêuticos, influentes divulgadores da informação médica, que até hoje visitam os mais remotos hospitais, clínicas e consultórios.

Tais propagandistas recebem treinamento de vendas específico sobre o medicamento que representam comercialmente, para serem capazes de persuadir os médicos de qualquer especialidade a adotá-los. Além de estarem capacitados a responder questões sobre o medicamento, trazem consigo referências científicas, artigos e pesquisas adicionais que reforçam sua credibilidade ${ }^{1}$.

Considerando as situações de interação propagandista-médico como um momento de atualização profissional, ou seja, de educação continuada, é possível afirmar que, apesar do evidente conflito de interesses inerente a essa modalidade de divulgação de conhecimentos científicos, é inegável que ela funcionou no passado e ainda funciona em algumas regiões do País, veiculando estas informações de modo exclusivo, em especial para quem não dispõe de outras fontes de consulta, nem internet.

Diante dessa realidade, é possível acreditar que esse espaço educativo se consolidou, historicamente, pela ausência de uma política pública que orientasse um programa governamental adequado, ágil e confiável de formação continuada para esses profissionais.

Desde então, com os avanços tecnológicos que possibilitaram a constituição daquela que hoje é definida como Sociedade do Conhecimento, o volume de informações científicas alcançou tal dimensão, que se torna impossível para um médico manter-se atualizado empregando apenas os mesmos recursos utilizados no passado, ou seja, propagandistas e eventos científicos. De modo geral, é inconcebível imaginar que a educação nos dias de hoje se dará apenas na escola, até os vinte ou trinta anos de idade. Sabe-se que o ser humano aprende por toda a vida, em situações de ensino formal ou informal, a partir de estudos independentes ou experiências vividas. Kenski afirma que nãoé possível considerar qualquer pessoa plenamente formada, qualquer que seja seu grau de instrução $0^{2,3}$. As demandas da sociedade contemporânea exigem que as instituições de ensino promovam constantes oportunidades para o desenvolvimento humano e profissional de seus cidadãos. É nesse contexto que se insere a educação continuada.
A veiculação da informação, por tantos meios diferentes e com tanta velocidade, pode gerar insegurança nos profissionais médicos, que têm dificuldade não só para acompanhar as mudanças, mas para selecionar as fontes confiáveis e as informações relevantes para efetivamente incorporá-las à sua prática de acordo com o contexto socioeconômico e cultural no qual trabalham.

A necessidade de um processo de formação continuada, regulado por organismos oficiais da área da medicina, como as associações de especialidades médicas e as universidades, se impõe agora mais do que nunca. No entanto, esta formação deverá considerar, além dos aspectos conceituais, aqueles relativos a procedimentos e atitudes, privilegiando a consolidação da ação profissional consciente e crítica. E esse processo requer a participação de outros profissionais formadores, além de médicos, especialistas e pesquisadores. Em especial, deve incluir um olhar para a prática pedagógica que o organiza e desenvolve, para as concepções de homem e educação que o informam, para as escolhas metodológicas que o orientam.

No intuito de contribuir para essa reflexão, este texto tem por objetivos retomar o processo de formalização da educação médica continuada a distância no Brasil em termos didático-pedagógicos e analisar algumas perspectivas dessas ações educativas.

\section{EDUCAÇÃO MÉDICA CONTINUADA: CONCEITOS E CARACTERÍSTICAS}

O termo Educação Médica Continuada (EMC), apesar de não encontrar correspondente exato no sistema educacional brasileiro oficial, tem se tornado cada vez mais familiar aos profissionais de medicina. Trata-se de um importante meio de educação permanente para o profissional do século 21, especialmente na área da saúde, onde o volume de informações científicas cresce exponencialmente. Deriva do conceito norte-americano de Continuing Medical Education (CME), um sistema de atualização profissional médica que oferece certificação oficial a partir de um número determinado de créditos, que podem ser obtidos por meio de atividades educacionais variadas, como o estudo de um artigo científico, a participação em eventos ou a realização de um curso completo.

Educação médica continuada consiste em atividades educacionais que servem para manter, desenvolver ou melhorar o conhecimento, habilidades, além de relacionamento e desempenho profissional que médicos utilizam para prover serviços a pacientes, ao público ou à profissão(tradução nossa $)^{4}$. 
O Accreditation Council for Continuing Medical Education (ACCME) é a organização norte-americana responsável pelos processos de desenvolvimento e promoção de padrões de qualidade para EMC, e por dirigir todo o processo de acreditação nacional. Além disso, estabelece critérios de avaliação, pelos quais o ACCME poderá conferir acreditação às instituições provedoras dos programas e atividades educacionais, presenciais ou a distância. É importante destacar que as atividades podem ter inúmeros formatos, desde participação em eventos científicos, discussões de casos clínicos, estudo de artigos, vídeos ou tutoriais, entre outros. Devem obedecer a um conjunto de elementos essenciais, que incluem a existência de um processo de planejamento que identifique necessidades e estabeleça metas de desempenho. Também recomenda cuidados especiais quanto a questões éticas e comerciais.

Desse modo, o conceito de acreditação, um neologismo com origem na língua inglesa, e de acordo com a própria ACCME: “É a decisão tomada pelo ACCME, ou por uma sociedade médica estadual reconhecida, de que uma organização atinge os requisitos para se tornar um provedor de CME, como definido pelo ACCME". Um termo de acreditação tem a duração padrão de quatro anos. O Conselho oferece um minucioso conjunto de requisitos para que uma organização se submeta à acreditação.

Na América Latina, podem ser encontradas outras regras e diferentes formas de organização da educação continuada para acreditação das especialidades médicas, que não são objeto de estudo deste texto. A título de ilustração, no Chile, o médico pode adquirir o grau de especialização em programas de postítulo ou postgrado e submeter-se à Calificación Médica Nacional e Exámen Médico Nacional (EMN) ${ }^{5}$.

A área de medicina no Brasil se diferencia das outras áreas profissionais por dispor de regulamentação específica para acreditação/validação. A Comissão Nacional de Acreditação (CNA), por meio da resolução n⿳0 1.772/2005, instituiu o Certificado de Atualização Profissional para os portadores dos títulos de especialista e certificados de áreas de atuação. Esse certificado tem a validade de cinco anos, após os quais os médicos deverão submeter-se obrigatoriamente a um processo de certificação de atualização profissional.

O processo de certificação de atualização profissional tem como objetivo manter, por meio de educação continuada, a qualificação permanente dos especialistas, buscando sua valorização profissional e, consequentemente, garantindo aos pacientes o atendimento adequado(p.3)
As atividades de atualização podem ser: participação em congressos, jornadas e simpósios ou em cursos na especialidade, desde que tenham aprovação da CNA. Eventos a distância somente terão validade se houver questionários de avaliação da aprendizagem.

Embora o escopo deste trabalho se concentre nos cursos voltados à atualização profissional, é importante lembrar que, no Brasil, o título de especialista é fornecido pelas sociedades médicas de cada especialidade após provas nacionais e tem regulamentação específica não estabelecida pelo MEC. Por exemplo, a Sociedade Brasileira de Endocrinologia e Metabologia ${ }^{7}$ estabelece em seu website as regras para o Concurso para Obtenção do Certificado de Área de Atuação em Endocrinologia Pediátrica de 2007.

A partir das determinações do Conselho de Acreditação para Educação Médica Continuada (Accreditation Council for Continuing Medical Education - ACCME) dos EUA, das determinações da Comissão Nacional de Acreditação (CNA), e das disposições do Ministério da Educação (MEC), ambos do Brasil, foi possível explicitar algumas das semelhanças observadas entre eles. Na ACCME, a acreditação é outorgada tanto ao provedor do curso quanto ao médico, não há modelo pedagógico definido, e os objetivos de ensino são "manter, desenvolver ou melhorar o conhecimento e/ou habilidades". OCNA não prevê a acreditação à instituição ofertante, pois esta deve ser necessariamente credenciada oficialmente. Seu objetivo é "manter a qualificação permanente dos especialistas", mas, assim como a ACCME, não oferece parâmetros pedagógicos para seus cursos, que valem créditos, assim como a participação em eventos. OMEC, por não estar relacionado especificamente com a acreditação médica, determina que um curso em nível de especialização deve ter no mínimo 360 horas e conter um projeto pedagógico.

\section{ASPECTOS DIDÁTICO-PEDAGÓGICOS DA EMC EM PAÍSES DESENVOLVIDOS}

É possível afirmar que a Educação Médica Continuada, assim como a Educação Médica Continuada a distância correspondem a ações educacionais. A Educação Médica Continuada a Distância pode ser definida como "um meio de acesso ao conhecimento e ao aperfeiçoamento dos profissionais e estudantes de Medicina, da área de saúde em geral e também do público leigo, para que possam adquirir novos conhecimentos" ${ }^{\prime 8}$, por meio das Tecnologias de Informação e Comunicação (TICs). Reúne as seguintes características: 
Quadro 1

Principais características de programas de EMC

\begin{tabular}{|l|l|l|l|}
\hline Características & \multicolumn{1}{|c|}{ ACCME - EUA } & MEC (lato sensu) - Brasil & CNA - Brasil \\
\hline Quem recebe a acreditação & O provedor do curso e o médico & $\begin{array}{l}\text { Não se aplica; é fornecido } \\
\text { certificado }\end{array}$ & O médico especialista \\
\hline Tipo de curso oferecido & $\begin{array}{l}\text { Sem modelo definido. Valem } \\
\text { diferentes tipos de atividades, } \\
\text { como eventos, discussões de caso } \\
\text { clínico, cursos, etc. }\end{array}$ & $\begin{array}{l}\text { Mínimo de } 360 \text { horas; tem projetogógico } \\
\text { pedaistema de créditos: } \\
\text { valem eventos e cursos }\end{array}$ & $\begin{array}{l}\text { Manter a qualificação } \\
\text { permanente dos } \\
\text { especialistas }\end{array}$ \\
\hline Objetivos de ensino & o conhecimento e/ou habilidades & $\begin{array}{l}\text { Variados, sempre em nível de } \\
\text { especialização, com caráter de } \\
\text { educação continuada }\end{array}$ \\
\hline
\end{tabular}

- Dirige-se à atualização e ao desenvolvimento profissional de médicos, em resposta às necessidades impostas pela Sociedade do Conhecimento;

- Utiliza o apoio das TICs para criar situações em que a distância transacional funciona em favor da autoaprendizagem e/ou da aprendizagem colaborativa;

- Considera as peculiaridades do aprendizado de adultos, ou andragogia;

- É estruturada a partir de uma concepção de educação, seja de forma consciente e explícita ou subjacente às atividades didáticas propostas.

Nos Estados Unidos, assim como em outros países desenvolvidos, esta modalidade de EMC já é bastante difundida. Em geral, corresponde a abordagens pedagógicas comuns naquele país, que não valorizam apenas a transmissão de informações, mas principalmente o desenvolvimento de habilidades e competências, com muita atenção às medidas de desempenho e à produtividade dos alunos. A quantidade de literatura disponível sobre avaliação de aprendizagem em educação continuada a distância produzida por autores norte-americanos demonstra a grande preocupação em fazer valer o custo dos esforços empregados para que um curso dessa natureza se realize e seja efetivo.

Bernard Sklar, da Universidade da Califórnia (São Francisco, EUA), que desde seu trabalho de mestrado em 2000 mantém um website atualizado em CME, lista mais de 300 websites, que oferecem cerca de 13 mil atividades e mais de 22 mil horas de EMC online naquele país. Ele não é explícito em relação à abordagem pedagógica adotada, porém seu relato sobre os recursos de ensino utilizados oferece fortes indícios de que grande parte dessas atividades decorre das concepções tradicional ou comporta- mentalista de educação. Os dados coletados por esse autor demonstram que os websites pesquisados oferecem:

- Textos e gráficos, em 38\% dos casos;

- Instrução por meio de textos, em 28\% deles;

- Instrução por meio de diapositivos com áudio, em 23\%;

- Possibilidades de interação, em 17\%;

- Diapositivos e vídeo, em 7\%;

- Roteiro de atividades, em 7\%;

- Instrução baseada em perguntas e respostas, em 4\%.

Ainda segundo Sklar, a maior parte deles não aproveita a oportunidade conferida pelo computador de empregar múltiplas possibilidades de aprender e de provocar outras formas de interação entre alunos e professores ${ }^{9}$.

Zimitat ${ }^{10}$ e McAdams ${ }^{11}$ concordam em que a maioria das atividades oferecidas na forma de EMC online atualmente está centrada na figura do instrutor/professor como responsável pela entrega de conteúdos e pela condução de métodos interativos de ensino. Fornece alguns exemplos, mencionando recursos audiovisuais, fóruns de discussão, grupos de bate-papo, pesquisa na internet, questionários interativos, simulações virtuais de pacientes e diagnósticos, estudos de caso progressivos, entre outros.

\section{EMC A DISTÂNCIA NO BRASIL: PERSPECTIVAS}

No Brasil, o impacto da EMC online ainda é muito pequeno, mas com grande potencial. A internet tem tido um enorme crescimento no País nos últimos anos, uma cifra que hoje ultrapassa 40 milhões de usuários, segundo pesquisa disponível no website do Instituto Brasileiro de Opinião Pública e Estatística (lbope), em $2008^{12}$. 
Este uso ainda está concentrado nas camadas socioeconômicas mais favorecidas, residentes nos grandes centros urbanos, onde se registram altos índices de problemas de trânsito e de violência que, de certo modo, impulsionam as formas de entretenimento e cultura domésticas, como a internet. Cabe ressaltar que apenas 29\% desses usuários acessam sites de Educação e Carreiras, segundo o Ibope.

Segundo o Anuário Brasileiro Estatístico de Educação Aberta e a Distância (Abraead), mais de dois terços de todas as instituições de ensino superior dos Estados Unidos adotam atualmente a educação online ${ }^{13}$. Além disso, o anuário informa que o número de alunos em cursos online cresce $18,2 \%$ ao ano. São programas formais e não formais, com milhões de estudantes, em sua grande maioria adultos em educação continuada, educação profissional ou pós-graduação. Tais cursos há muito são oferecidos na modalidade presencial. Entretanto, com o advento das novas tecnologias, a educação online passou a ser bastante atrativa para conduzir tais iniciativas.

No Brasil, ainda de acordo com o Abraead, mais de 1,2 milhão de pessoas estudaram a distância em 2006. No nível de pós-graduação lato sensu, o que mais tem crescido, o número de cursos saltou de 246 em 2006 para 404 em 2007. Os dados sobre 2008 ainda não estão disponíveis, mas a tendência de crescimento persiste. Há que se mencionar também a Universidade Aberta do Brasil (UAB), resultante da articulação e integração de instituições de ensino superior, municípios e estados, nos termos do artigo 81 da Lei de Diretrizes e Bases da Educação, que visa à democratização, expansão e interiorização da oferta de ensino superior público e gratuito no País ${ }^{14}$.

Recentemente, foram divulgadas informações de que o Ministério da Educação (MEC) pretende suprimir a exigência de que as universidades mantenham um polo de apoio com sala de informática, biblioteca e tutor presencial nas cidades onde se oferecem cursos de pós-graduação lato sensu a distância, diminuindo as exigências e facilitando a expansão da educação a distância $(\mathrm{EaD})^{15}$. Ainda assim, a educação médica online oferecida por universidades é limitada quando comparada a outras áreas de conhecimento, particularmente a de educação, que foi alvo de iniciativas recentes dos governos federal e estadual no sentido de capacitar professores de ensino fundamental e médio, além das áreas de administração e tecnologia.

Entretanto, em função do dispositivo legal no $1.772 / 05$, emitido pelo Conselho Federal de Medicina, é provável que ocorram mudanças nessa situação. Este texto legal determina que os títulos de especialista, assim como outros certificados na educação médica profissional, terão seu período de validade reduzido para cinco anos, após os quais os médicos terão que se submeter a um novo processo de validação ou acreditação desse título. Considerando os argumentos mencionados sobre os aspectos práticos para a utilização da $\mathrm{EaD}$, é possível que se desenhem renovadas oportunidades para cursos online nessa área.

Tendo em vista as dimensões continentais do Brasil e seu histórico de dificuldades sociais, educacionais e econômicas, a educação continuada a distância é certamente um recurso muito importante para responder à demanda de atualizar profissionais de todas as áreas. Em especial na área da saúde, a possibilidade de divulgar o conhecimento produzido nos grandes centros para profissionais das áreas mais remotas figura como uma das mais importantes conquistas da $\mathrm{EaD}^{16}$.

Mesmo para os profissionais que vivem nas grandes cidades, a EaD pode oferecer vantagens, uma vez que em função de variadas demandas eles têm tido cada vez menos disponibilidade de tempo para frequentar cursos presenciais, que ocorrem, em geral, em espaços e horários determinados. Isso é especialmente relevante em relação aos médicos, comprometidos com mais de uma jornada de trabalho, com plantões noturnos em hospitais ou em atendimento a chamadas de emergência. Nesses casos, a EaD pode ser uma alternativa para a atualização desses profissionais, sem oferecer restrições de tempo (horários flexíveis) ou de espaço.

\section{CONSIDERAÇÕES FINAIS: UM OLHAR PEDAGÓGICO}

Contando com a necessária expansão da Educação Médica Continuada, em especial na modalidade a distância, no Brasil, será preciso dedicar atenção também a alguns aspectos de cunho pedagógico.

No Brasil, a Comissão Nacional de Acreditação (CNA) prevê que as atividades educacionais podem ser realizadas por meio de programas de educação a distância. E estabelece, como único critério pedagógico para a concessão de créditos, a realização de uma avaliação de desempenho do participante, embora não determine regras ou critérios para essa avaliação, mesmo em se tratando de cursos completos. Desta forma, a legislação não faz restrições sobre a ferramenta de avaliação de desempenho, que pode ser apenas um questionário de múltipla escolha com resposta automática, no qual o aluno tem acesso à resposta correta imediatamente. Também permite que o aluno realize o exame mais de uma vez, o que pode limitar a medida objetiva de sua aprendizagem ${ }^{6}$.

Atualmente, várias instituições públicas e privadas já oferecem EMC a distância. Por exemplo, as associações médicas esta- 
duais e nacionais, que disponibilizam aos seus associados e demais profissionais de saúde programas de atualização profissional a distância. Esses cursos são produzidos por instituições privadas de ensino e de pesquisa e utilizam diversas tecnologias, como Internet e televisão via satélite.

A análise de alguns desses programas permite supor que a concepção de educação que os informa seja a tradicional, baseada na transmissão de informações. Tais programas, em especial os televisivos, poderiam ter sua utilização enriquecida se transmitidos em sessões agendadas para grupos de médicos, seguidas de discussões presenciais ou virtuais, síncronas ou assíncronas.

No entanto, não foi possível identificar com clareza os critérios de avaliação e de aproveitamento ou a metodologia de ensino utilizados nesses programas, o que pode sugerir desconhecimento pedagógico ou descompromisso com a formação profissional, por parte dos autores, no processo de elaboração e condução da ação educativa. Mesmo tais programas sendo baseados no modelo tradicional de educação, essas lacunas não se justificam, pois as faculdades e cursos de Medicina brasileiros sempre se orgulharam do alto nível de exigência imposto aos alunos.

Foi possível também observar que a maior parte dos cursos explicita os objetivos de ensino sob a forma de relação dos conteúdos a serem abordados, ao invés de descrever as transformações conceituais, procedimentais e atitudinais que o professor se propõe a alcançar em relação a seus alunos. Isso pode revelar a ausência de uma concepção de educação no planejamento do curso.

Esse aspecto pode tornar-se particularmente relevante se considerado o perfil do professor de ensino superior, no Brasil, que recebe a atribuição de planejar e conduzir cursos a distância. Trata-se, em geral, de um profissional com títulos de pós-graduação na área de conhecimento do curso a ser ministrado, porém sem formação pedagógica adequada para atuar em cursos tanto presenciais quanto a distância. Vale lembrar também que nem sempre a EMC a distância é oferecida por instituições educacionais, como no caso daquelas propostas pelas sociedades médicas, que oferecem acreditação a médicos especialistas e nem sempre têm formato de curso. Às vezes, acontecem sob a forma de grupo de estudos, discussão de caso clínico ou de artigo científico online, por exemplo.

Para fazer frente às iniciativas de comercialização da EMC, a Associação Médica Brasileira (AMB) e o Conselho Federal de Medicina (CFM) se preparam para lançar o Programa Nacional de Educação Continuada a Distância para médicos. É gratuito e visa disseminar o conhecimento e as novas descobertas diagnós- ticas e terapêuticas, contribuindo, assim, para elevar o nível da medicina e ainda para a melhoria do atendimento aos pacientes. As aulas abrangerão as 56 especialidades médicas reconhecidas oficialmente pela $\mathrm{AMB}$ e pelo $\mathrm{CFM}^{17}$.

Na Universidade Federal de São Paulo (Unifesp) ${ }^{18}$, consolidada pela tradição em cursos de graduação e pós-graduação exclusivamente na área de saúde, a Educação a Distância é desenvolvida e administrada pelo Departamento de Informática em Saúde (DIS), que oferece programas de educação em saúde para leigos e para formação continuada do profissional de saúde, além da capacitação docente para a utilização de ambientes virtuais como recurso de apoio ao ensino presencial na graduação e pós-graduação ${ }^{19}$.

Para isso, conta com uma equipe multidisciplinar composta das áreas gerencial, tecnológica, de design e também de uma equipe de educação, responsável pela orientação pedagógica e pelo suporte aos docentes nos processos de planejamento de ensino, de desenvolvimento e avaliação dos cursos a distância.

De modo geral, essa equipe tem observado que muitos docentes, especialistas na área de saúde, priorizam os conteúdos a serem transmitidos, a qualidade estética e a sofisticação tecnológica, porém dedicam pouca atenção a aspectos pedagógicos, como definição de objetivos de ensino pautados no desenvolvimento de habilidades e competências ou na seleção de procedimentos de ensino que provoquem situações de aprendizagem significativa, reflexiva e crítica, como preconizado nas diretrizes curriculares para o ensino superior ${ }^{20}$ e pelos referenciais de qualidade para educação superior a distância, que definem que um curso a distância:

[...] deve apresentar claramente sua opção epistemológica de educação, de currículo, de ensino, de aprendizagem, de perfil do estudante que deseja formar; com definição, a partir dessa opção, de como se desenvolverão os processos de produção do material didático, de tutoria, de comunicação e de avaliação, delineando princípios e diretrizes que alicerçarão o desenvolvimento do processo de ensino e aprendizagem (SEED/MEC, p. 8-9) ${ }^{21}$.

Além disso, esses referenciais ainda recomendam que:

[...] o uso inovador da tecnologia aplicado à educação, [...] e mais especificamente, à educação a distância deve estar apoiado em uma filosofia de aprendizagem que proporcione aos estudantes a oportunidade de interagir, de desenvolver projetos compartilhados, de reconhecer e respeitar diferentes culturas e de construir o conhecimento (SEED/MEC, p. 8-9) 22 . 
No âmbito da formação em saúde, o desconhecimento das características pedagógicas inerentes aos processos educativos pode ser explicado pela falta de formação específica deste profissional para a atividade educativa e pela intensa familiaridade com os modelos de ensino tradicional presencial, centrados na figura do professor detentor do conhecimento, ainda em vigor. O bom desempenho do professor tem sido historicamente avaliado pelos conhecimentos técnicos, não se questionando a competência didático-pedagógica desse profissional ${ }^{23}$. No entanto, segundo Moreno, “a complexidade dos processos de aprendizagem tem demandado, dos professores, um enfoque mais construtivo, desvelando os movimentos de apropriação e produção dos conhecimentos trabalhados em espaços escolares e acadêmicos"'(p. 455) ${ }^{24}$.

E essa consideração crítica vale tanto para os professores responsáveis pelo conteúdo específico de saúde como para a equipe de desenvolvedores dos cursos do DIS, que foi inicialmente formada por profissionais das áreas tecnológica e biológica. Com a inclusão de profissionais de educação na equipe, as questões referentes ao diálogo entre essas três áreas do conhecimento - saúde, tecnologia e educação - em favor da maior consistência das propostas pedagógicas passaram a ser explicitadas e vêm sendo enfrentadas com relativo sucesso ${ }^{25}$.

Diante dessas dificuldades, é possível considerar que não basta ter acesso ao repertório de conhecimentos científicos, nem usar recursos tecnológicos avançados, nem mesmo atender ao alto grau de exigência do profissional de saúde, em geral médicos familiarizados com a qualidade dos serviços prestados. Nada disso isoladamente pode garantir que a proposta pedagógica de um curso de educação continuada online esteja adequada às necessidades do profissional médico no mundo contemporâneo.

De outro lado, ainda é possível supor que os médicos-alunos desses cursos esperam que eles reproduzam os processos educativos dos quais foram sujeitos durante sua formação profissional, isto é, de transmissão e reprodução de informações. Parecem não desejar ampliar o escopo da formação continuada para incluir aspectos psicossociais ou éticos, desestimulando o investimento em abordagens pedagógicas diferenciadas e dificultando alterações significativas no modelo pedagógico vigente.

No entanto, em face das mudanças sociais, muitas transformações são previstas na educação superior. A realidade vem impondo que as ações educativas tenham em vista a construção reflexiva do conhecimento, ao invés de sua mera reprodução, como praticado até hoje.
E essa inquietação não é exclusiva do sistema educacional brasileiro. Ela também pode ser observada em outros países da América do Sul. De acordo com Armengol(p.21) ${ }^{26}$ :

Los procesos actuales de transformación buscan superar la concepción de la educación como simple "transmisión-acumulación" de conocimientos e información. De esta manera, la llamada "crisis educativa" es en buena parte una crisis del modelo pedagógico tradicional.

Mesmo nos países desenvolvidos, registram-se críticas ao modelo educacional vigente que devem ser observadas criteriosamente. Peterson ${ }^{27}$, em seu trabalho sobre o estado da arte da EMC na internet em 2005 nos Estados Unidos, mostra que websites patrocinados por universidades geralmente forneciam informações sobre EMC seguidas de links para os sites comerciais onde esses cursos eram oferecidos. Observa ainda que o número de ações de EMC online oferecido por sites comerciais era maior do que o oferecido pelas universidades e que isso já havia sido observado em sua pesquisa anterior, em 1998.

O autor também considera que muitos desses sites comerciais que ofereciam EMC online não atendiam a padrões mínimos de qualidade e que as universidades, que potencialmente teriam condições de garantir tal qualidade, desconsideravam este espaço de oferta de cursos.

Essa tendência de as instituições privadas oferecerem educação continuada a distância também pode ser observada na América Latina.

A Sociedade do Conhecimento impõe a formação inicial e continuada de profissionais e cidadãos com um novo conjunto de competências para atuar com eficiência e responsabilidade. É preciso procurar integrar e propor abordagens pedagógicas que efetivamente valorizem, além dos conteúdos de ensino, a disposição para a pesquisa, a autonomia na busca da informação, o espírito colaborativoe a postura ética, entre outras.

Nesse sentido, cabe à EMC no Brasil propor ações que visem:

- Atualizar profissionais em coerência com o perfil descrito pelas Diretrizes Curriculares Nacionais do curso de Medicina e do Sistema Único de Saúde;

- Criar condições que favoreçam a aquisição de novas competências procedimentais, atitudinais e conceituais derivadas do uso das novas tecnologias de informação e comunicação;

- Avaliar o desempenho de suas ações educativas continuamente, com enfoque formativo;

- Considerar o perfil do aluno, ou seja, do médico formado no sistema educacional brasileiro, que geralmente não possui habilidades de busca e construção autônoma do conhecimento. 


\section{REFERÊNCIAS}

1. Pedrosa R, Duarte Z. O processamento da informação e sua recepção pelo propagandista de produtos farmacêuticos e pelo especialista em ginecologia e obstetrícia. $7^{\circ}$ Cinform - Encontro Nacional de Ensino e Pesquisa da Informação, 2007; Salvador, BA. Salvador: Instituto de Ciência da Informação da UFBA; 2007.

2. Kenski VM. Tecnologias e ensino presencial e a distância. 2a ed. Campinas: Papirus; 2003. 157p.

3. Oliveira MAN. Educação à Distância como estratégia para a educação permanente em saúde: possibilidades e desafios. Rev Bras Enferm. [periódico na Internet]. 2007 [acesso em 27 abr. 2009]; 60(5):585-589. Disponível em: http:/ / www.scielo.br/scielo.php?script=sci_arttext\&pid=S0034-71672007000500019

4. Accreditation Council for Continuing Medical Education [homepage]. Chicago, IL: ACCME; c2009- [cited 20096 feb.]. Available from: http://www.accme.org

5. Espinoza G Ricardo. Examen Médico Nacional y Médica en Chile. Rev Chil Pediatr. [periódico na Internet]. 2008 [acesso em 6 fev. 2009]; 79(1):9-12. Disponível em: http:/ / www.scielo.cl/scielo.php?script=sci_arttext\&pid=S0370-41062008000100001\&lng=es\&nrm=iso\#back

6. Brasil. Conselho Federal de Medicina. Resolução CFM n⿳‥ 1772, de 12 agosto de 2005. Institui o Certificado de Atualização Profissional para os portadores dos títulos de especialista e certificados de áreas de atuação e cria a Comissão Nacional de Acreditação para elaborar normas e regulamentos para este fim, além de coordenar a emissão desses certificados. Revoga a Resolução CFMn. 1.755/2004. Diário Oficial da União [online] Brasília, 12 ago. 2005; Seção 1, p.141-142. [acesso em 6 fev. 2009] Disponível em: $<$ http://www.smp.org.br/jornal/downloads/Resolucao_CFM_1772.pdf

7. Sociedade Brasileira de Endocrinologia e Metabologia [homepage]. Rio de Janeiro: SBEM; c2009- [acesso em 6 fev. 2009]. Disponível em: http:/ /www.endocrino.org.br

8. Associação Paulista de Medicina [homepage]. São Paulo: APM; c2008 - [acesso em 6 fev. 2009]. Educação Médica. Educação Médica Continuada; [aproximadamente 1 tela]. Disponível em: http://www.apm.org.br/aberto/educacaomedica_interna.aspx?id=121

9. Sklar BM. The current status of online continuing medical education [online]. San Francisco; 2000. [Thesis] - Califórnia University. Medical Information Science. [cited 20096 fev]. Available from: http://www.cmelist.com/mastersthesis

10. Zimitat C. Designing effective on-line continuing medical education. Med Teach [journal Internet]. 2001 mar [cited 20096 fev];23(2):117-22. Available from: http://www.ingentaconnect.com/content/apl/cmte/2001/00000023/00000002/art00001

11. Mc Adams S. The evolution of on-line CME: improved course delivery provides greater opportunity to participative in distance learning. Ont Med Rev [periodico na Internet]. 2002 abr [cited 20096 fev];69(4):[2 telas]. Available from:

http:/ /www.oma.org/pcomm/omr/apr/02ww.htm

12. Instituto Brasileiro de Opinião Pública e Estatística. Brasil superou o número de 40 milhões de pessoas com acesso à internet. Ibope [online]. 27 jun. 2008 [acesso em 13 mar. 2009]; Notícias. Press-releases: [aproximadamente 2 p.]. Disponível em: http://www.ibope.com.br/calandraWeb/servlet $/$ CalandraRedirect?tem $p=5 \&$ proj=PortalIBOPE\&pub $=\mathrm{T} \& \mathrm{db}=\mathrm{caldb} \& \mathrm{comp}=\mathrm{Not} \%$ EDcias\&docid=F0BA65FF8A513A48832574750050527E

13. Instituto Monitor. Anuário brasileiro estatístico de educação aberta e a distância. São Paulo: o Instituto;2006

14. Ministério da Educação. Coordenação de Aperfeiçoamento de Pessoal de Nível Superior [homepage]. Brasília: MEC;CAPES; c2006 - [acesso em 6 fev. 2009]. Universidade Aberta do Brasil. Disponível em: http:/ / www.uab.mec.gov.br

15. Takahashi F. Governo facilita curso de pós à distância. Folha de São Paulo [online]. 13 dez. 2007 [acesso em 13 mar. 2009]; Educação: [aproximadamente 2 p.]. Disponível em: http:/ / www1.folha.uol.com.br/folha/educacao/ult305u354491.shtml

16. Christante L, Ramos MP, Bessa R, Sigulem D. O papel do ensino a distância na educação médica continuada: uma análise crítica Rev Assoc Med Bras [periódico na Internet]. 2003 [acesso em 6 fev 2009];49(3): [aproximadamente 8 p.]. Disponível em: http://www.scielo.br/pdf/ramb/v49n3/a39v49n3.pdf

17. Associação Médica Brasileira [homepage]. São Paulo: AMB; c2008 - [acesso em 6 fev. 2009]. Disponível em: http:/ / www.amb.org.br/

18. Universidade Federal de São Paulo [homepage]. São Paulo: UNIFESP; c2006-2008 [acesso 6 fev. 2009]. Disponível em: http://www.unifesp.br

19. Leite MTM. Cursos em educação médica continuada on-line na América Latina: um olhar pedagógico. São Pau- 
lo; 2007. Mestrado [Dissertação] - Universidade Federal de São Paulo.

20. Brasil. Ministério da Educação. Conselho Nacional de Educação. Câmara de Educação Superior. Resolução CNE/CES nº. 4 de 7 de novembro de 2001. Institui diretrizes curriculares nacionais do curso de graduação em medicina. Brasília, DF: Diário Oficial da União. Brasília, 9 nov. 2001; Seção 1, p.38. [acesso em 6 fev. 2009]. Disponível em: http://portal.mec.gov.br/sesu/arquivos/pdf/0401Medicina.pdf

21. Ministério da Educação. Secretaria de Educação à Distância [homepage]. Brasília: MEC; c2008 - [acesso em 6 fev. 2009]. Secretarias. Secretaria de Educação a Distância. Legislação. Referenciais de qualidade para EAD. Disponível em: http://portal.mec.gov.br/seed/index.php?option $=$ com_content\&task=view\&id $=62$

22. Ministério da Educação. Secretaria de Educação a Distância. Referenciais de qualidade de educação superior e a distância. Brasília: MEC; 2007. 31p.

23. Zeferino AMB, Barros Filho A. A Capacitação docente: experiência da pós-graduação em saúde da criança e do adolescente com a disciplina Pedagogia Médica e Didática Especial na FCM/UNICAMP. In: Batista NA, Batista SHS. Docência em Saúde: temas e experiências. São Paulo: SENAC; 2004. p. 225-231.

24. Ruiz-Moreno L, Sonzogno MC, Batista SHS, Batista NA. Concept map: testing analytical criteria. Ciênc Educ (Bauru) [periódico na Internet]. 2007 [acesso em 12 maio 2009];23(3):453-63. Disponivel em: http://www.scielo.br/scielo.php?script=sci_arttext\&pid=S1516-73132007000300012\&lng=en\&nrm=iso.
25. Leite MTM. Relato de experiência: oficinas Moodle para docentes da UNIFESP. [pôster apresentado no $13^{\circ}$ Congresso Internacional de Educação a Distância; 2007; Curitiba: ABED; 2007].

26. Armengol MC. Universidad a distancia y la transformación de la sociedad latinoamericana. [Artigo apresentado no $12^{\circ}$ Congresso Internacional de Educação a Distância; 2004; São Paulo: ABED; 2004].

27. Peterson MW. Continuing medical education on the internet: state of the art. J Contin Educ Health Prof [periodico na Internet]. 2005 [acesso em 20096 feb];19(4):242-9. Available from: http://www3.interscience.wiley.com/journal $/ 110471378 /$ abstract?CRETRY $=1 \&$ SRETRY $=0$

\section{CONTRIBUIÇÃO DOS AUTORES}

Maria Teresa Meirelles Leite: (autoria principal) concepção, elaboração da pesquisa e redação do artigo. Alda Luiza Carlini; Co-orientação, em especial aos temas relativos a teorias na área de educação e à condução da pesquisa. Monica Parente Ramos: revisão do texto e da formatação, debatedora sobre o trabalho. Daniel Sigulem: Orientação, em especial nos temas relativos à tecnologia e à saúde.

\section{CONFLITO DE INTERESSES}

Declarou não haver

\section{ENDEREÇO PARA CORRESPONDÊNCIA}

Maria Teresa Meirelles Leite

Rua Monte Alegre, 791 apto 121

Perdizes - São Paulo

CEP. 05014-000 SP 